\title{
Formação e Atuação de Psicólogos da Rede de Atenção Oncológica de Belo Horizonte/MG: Desafios e Perspectivas
}

\author{
Suellen Santos Lima de Almeida ${ }^{* \mathrm{a}}$, Alberto Mesaque Martins ${ }^{\mathrm{a}}$ Andréa Pereira Gazzinelli ${ }^{\mathrm{b}}$, \\ Virgínia Torres Schall ${ }^{\mathrm{a}} \&$ Celina Maria Modena ${ }^{\mathrm{a}}$ \\ ${ }^{\mathrm{a}}$ Fundação Oswaldo Cruz, Belo Horizonte, Brasil \& ${ }^{\mathrm{b}}$ Hospital Mário Penna, Belo Horizonte, Brasil
}

\begin{abstract}
RESUMO
A incidência do câncer e as novas formas de assistência e tratamento da doença convocam o psicólogo, enquanto profissional da saúde, a desenvolver estratégias que deem suporte à escuta do processo saúde-doença, na sua singularidade em diferentes contextos. Assim, foi objetivo deste estudo conhecer a formação e a atuação dos psicólogos da rede de atenção oncológica de Belo Horizonte Minas Gerais. Foram entrevistados 13 profissionais de instituições públicas e privadas. Os resultados apresentam a necessidade de uma formação que contemple a realidade dos serviços de saúde conforme proposto pelas políticas públicas numa perspectiva coletiva e social. Os discursos dos sujeitos apontam para os limites e possibilidades na construção de novos saberes e fazeres nas intervenções no contexto hospitalar.
\end{abstract}

Palavras-chave: formação do psicólogo; atuação do psicólogo; câncer.

\section{ABSTRACT \\ Education and Practice of the Psychologists' Network of Oncological Care of Belo Horizonte - MG: Challenges and Prospects}

The incidence of cancer and new forms of care and treatment of this disease invite the psychologist as a health professional to develop strategies that support the listening of the health-disease process in its uniqueness in different contexts. Thus, the aim of this study was to investigate the formation and the performance of the psychologists' oncology network in Belo Horizonte - Minas Gerais. We interviewed 13 professionals from public and private institutions. The results pointed to the need for a formation that addresses the reality of health services as proposed by public policies, in a social and collective perspective. The speech of the participants point to the limits and possibilities for the construction of new knowledge and practices in interventions at the hospital context.

Keywords: training of psychologists; practice; cancer.

O câncer tem sido considerado um problema de saúde coletiva, atingindo indivíduos de todas as idades, gêneros e classes sociais. Segundo a Organização Mundial da Saúde (WHO, 2009), para o ano de 2030 são esperados cerca de 27 milhões de casos novos de câncer e 17 milhões de óbitos, além de 75 milhões de pessoas que estarão convivendo, anualmente, com o câncer. No Brasil, as estimativas do Instituto Nacional do Câncer (INCA) para o ano de 2012 que também são válidas para o ano de 2013, apontam para uma incidência de 518.510 casos (INCA, 2011).
Apesar do desenvolvimento dos conhecimentos da ciência e do desenvolvimento de tecnologias para o diagnóstico, controle e prevenção do câncer, a percepção sobre a enfermidade ainda traz o medo em seu cerne (Teixeira \& Fonseca, 2007). O estigma que perpassa o imaginário social concebe a doença como um dos símbolos do sofrimento e da morte, seja por quem já padeceu por essa doença ou teve algum ente querido afetado, bem como por aqueles que nunca tiveram contato com o câncer (Barbosa \& Francisco, 2007; Doro, Pasquin, Medeiros, Bittencourt \& Moura,

Endereço para correspondência: Suellen Santos Lima de Almeida - suellen@cpqrr.fiocruz.br 
2004; Rey, 2006; Silva, 2006). Considerando este cenário, o psicólogo tem sido convocado a trabalhar na oncologia tanto na perspectiva hospitalar, quanto ao nível ambulatorial, pois ao atuar no campo da saúde, o psicólogo possibilita a construção de espaços de escuta e de elaboração do processo de adoecimento para o paciente, sua família e os profissionais de saúde (Angerami-Camon, 2001; Spink, 2003).

A possibilidade de promover um olhar diferenciado do modelo biomédico, somada a uma escuta atenta, coloca o psicólogo na posição de um mediador e catalisador das relações interpessoais existentes neste contexto (Andreoli, 2008). Dessa forma, ao promover uma atitude compreensiva este profissional auxilia pacientes, familiares e demais profissionais a expressarem suas dificuldades, podendo repercutir favoravelmente nos resultados do tratamento e, consequentemente na qualidade de vida dos envolvidos (Karam, Guimaro \& Rodrigues, 2008). O constante contato com a fragilidade humana e com os sentimentos associados ao adoecer, são experiências vividas no cotidiano dos profissionais de saúde, sobretudo no âmbito da oncologia (Silva, 2009). Por outro lado, ainda persistem propostas de formação em saúde centradas exclusivamente na cura dos pacientes, contribuindo para o sentimento de frustração profissional quando a mesma não se concretiza (Santos, 2003). Na tentativa de minimizar o sofrimento dos profissionais, frente a esta questão, Junqueira e Kóvacs (2008), defendem a inclusão da temática morte na formação dos profissionais de saúde e Silva (2010), ressalta a criação de grupos de escuta visando compartilhar, acolher e elaborar o sofrimento, como alternativa para se trabalhar, não só com a morte, mas também com cuidados paliativos de forma integral.

Nos últimos anos, tem-se discutido a questão da formação do profissional de saúde para o trabalho na oncologia. A formação em psicologia para atuar na assistência às pessoas com câncer deve fornecer subsídios para o trabalho em programas de prevenção e no tratamento das pessoas com câncer, considerando-se as informações, crenças e atitudes dos sujeitos envolvidos (Kóvacs, Macieira \& Carvalho, 2008). Assim, foi objetivo deste trabalho conhecer a formação e a atuação dos psicólogos da rede oncológica de Belo Horizonte.

\section{MÉTODO}

O estudo foi realizado na perspectiva da pesquisa qualitativa (Minayo, 2007), tendo em vista o seu potencial para subsidiar a compreensão das crenças, representações, sentimentos, sentidos e significados que os psicólogos atribuem à sua formação e às práxis nos serviços de oncologia. Nesse sentido, foram realizadas entrevistas semiestruturadas com psicólogos que atuam nos serviços especializados em oncologia, públicos e privados, situados na cidade de Belo Horizonte. As entrevistas estiveram alicerçadas no perfil acadêmico; concepções acerca da formação e nas práticas desenvolvidas no âmbito da oncologia. Os discursos foram gravados, transcritos e analisados na perspectiva da Análise de Conteúdo (Bardin, 1977). Definida como um conjunto de técnicas de análise das comunicações, a análise de conteúdo utiliza de procedimentos sistemáticos e objetivos do conteúdo das mensagens.

A partir de consulta à página virtual do Cadastro Nacional de Estabelecimento em Saúde do Ministério da Saúde (CNES) (2011), foram identificados os serviços especializados em oncologia, públicos e privados, situados na capital mineira. Em seguida, foi realizado contato telefônico, ou via correio eletrônico convidando todos os psicólogos que atuam nos serviços de psicologia dessas instituições para participação no estudo.

Os entrevistados foram esclarecidos sobre a confidencialidade das informações prestadas e, visando garantir o anonimato, tiveram seus nomes substituídos por nomes com a letra $\mathrm{P}$, remetendo à inicial da profissão. $\mathrm{O}$ projeto foi submetido e aprovado pelo Comitê de Ética em Pesquisa do Centro de Pesquisas René Rachou Fundação Oswaldo Cruz (parecer 13/2010) em consonância à Resolução 196/96, que dispõe sobre Diretrizes para Pesquisas com Seres Humanos no Brasil do Conselho Nacional de Saúde.

Após análise das entrevistas, puderam-se estabelecer os seguintes eixos temáticos: a formação para atuação na área da saúde, a práxis visando atender as demandas e as dificuldades institucionais que perpassam o fazer do psicólogo.

\section{RESULTADOS E DISCUSSÃO}

\section{A busca por conhecimentos para atuar na saúde}

A consulta ao CNES apontou para 23 serviços especializados em oncologia localizados na cidade de

Interação Psicol., Curitiba, v. 17, n. 1, p. 91-98, jan./abr. 2013 
Belo Horizonte. Destes, (39\%) não possuíam, no momento da pesquisa, psicólogos em seu quadro de profissionais, e (8\%) mantém um serviço terceirizado de psicologia para atendimento de seus pacientes. Estes dados parecem refletir as dificuldades de inserção do psicólogo na rede de atenção oncológica e ainda uma possível terceirização do trabalho destes profissionais, não atendendo ao disposto na Portaria $\mathrm{n}^{\circ} 3.535 / 1998$, do Ministério da Saúde, que orienta a inserção de psicólogos nos serviços de oncologia (Brasil, 1998).

Aceitaram o convite para participação na pesquisa, 13 psicólogos que trabalham em oito das instituições, sendo três privadas e cinco públicas. Os hospitais públicos caracterizaram-se como beneficentes (3), universitário (1) e pertencente à rede da Fundação Hospitalar do Estado de Minas Gerais (FHEMIG) (1), segundo classificação do CNES.

Dos entrevistados, $92,4 \%$ são do sexo feminino, corroborando estudos anteriores que evidenciam a maior presença das mulheres nos serviços de saúde (Spink, 2006; CFP, 2010). Tais dados apresentam a Psicologia como uma profissão essencialmente feminina no Brasil (Bastos, Gondin \& Borges-Andrade, 2010). Castro e Yamammoto (1998) ressaltam que enquanto os homens ocupam as áreas mais tradicionais da profissão, como a clínica e o trabalho, as mulheres predominam nas áreas emergentes como a psicologia hospitalar.

Apesar de apresentarem um tempo de formação que varia de 2 a 34 anos, os profissionais de psicologia entrevistados, relataram dificuldades em sua atuação no campo da saúde e atribuem isso à sua formação: "naquela época não se falava em psicologia hospitalar dentro das faculdades" (Paula). Silva (1992), ao examinar a formação do psicólogo para atuar na saúde pública aponta para uma defasagem entre os conteúdos da formação universitária e as necessidades deste setor. Para a autora, a universidade não está adequando a formação do graduando para as reais necessidades que a população apresenta.

Nessa mesma direção, outras pesquisas têm demonstrado que a academia não possibilita ao profissional uma formação adequada à intervenção em espaços territoriais que demandam alto grau de resposta/ação como é o caso dos hospitais (Dimenstein, 2001, Spink, Bernardes \& Menegon, 2006). A formação do psicólogo, ao direcionar sua prática para atuações limitadas no setor da saúde, torna-se de certa forma, corresponsável pelas dificuldades enfrentadas pelos profissionais em lidar com a demanda das instituições de saúde e dos usuários, bem como de adaptar-se ao perfil profissional exigido pelo SUS (Dimenstein, 2001).

A análise do currículo dos cursos de graduação em Psicologia aponta para uma valorização da doença e das técnicas em detrimento da escuta da história de vida dos sujeitos. Assim, a proposta do SUS em privilegiar a promoção das condições de vida e a compreensão do sujeito dentro de seu contexto histórico, político, social e cultural é suprimida pela ênfase dada as técnicas de intervenção e a preocupação entre o que é normal ou patológico (Guareschi, Dhein, Reis, Machry \& Bennemann, 2010). Além disso, segundo Kind e Coimbra (2011), alunos e professores apresentam-se encastelados na produção do conhecimento sem contato com a realidade dos serviços de saúde pública, o que os fazem ficar alheios ao cotidiano das práticas.

Carvalho e Cecim (2006) apontam que os profissionais de saúde ainda possuem uma formação centrada em conteúdos que não possibilitam uma orientação integradora entre ensino e trabalho. A trajetória dos psicólogos entrevistados indica que o ingresso na área hospitalar, ainda durante a graduação, nas atividades de estágios curriculares ou não curriculares, constituiu uma alternativa para a superação das dificuldades de articulação entre teoria e prática, além de ser a porta de entrada para o mercado de trabalho: "antes de me formar já havia feito estágio durante um ano no hospital X, com pacientes de gestação de alto risco. Foi ali que ingressei na área hospitalar" (Paula).

Em sua $13^{a}$ edição a Conferência Nacional de Saúde (2008) ao discutir os meios de integração ensinoserviço, sinaliza a importância dos intercâmbios, convênios, parcerias, integrações e articulações entre as instituições de ensino e a rede de serviços do SUS. Entre as atividades mais citadas para essa integração estão os estágios (Batista \& Gonçalves, 2011).

Mais do que a associação da teoria com a prática, o estágio possibilita a construção da identidade profissional, o desenvolvimento das estratégias de enfrentamento aos estressores das profissões do campo da saúde, além de promover a vivência de problemas e dificuldades da prática profissional, o que pode ser abordado em sala de aula de maneira superficial (Rudnicki \& Carlloto, 2007). A utilização de metodologias de ensino-aprendizagem que possibilitem aos graduandos construções de conhecimentos a partir dos problemas da realidade, visando assim, a integração 
entre teoria e prática é uma das estratégias pensadas por Feuerwerker (2003) para a superação da defasagem entre o que é aprendido nas universidades e o que é demandado pelo sistema de saúde vigente.

$\mathrm{Na}$ tentativa de superar o abismo entre as demandas reais da saúde e a formação dos profissionais os Ministérios da Saúde e da Educação, construíram e implantaram propostas como o Programa Nacional de Reorientação da Formação Profissional em Saúde PRÓ-Saúde (Brasil, 2007) e o Programa de Educação pelo Trabalho para a Saúde - PET-Saúde (Brasil, 2008) que, por meio de parcerias entre universidadeserviço-comunidade, buscam desenvolver ações que valorizem a formação de profissionais atentos à realidade do país, bem como a modificação curricular para que atenda às necessidades do SUS (Romagnoli, 2006).

Uma vez que a maioria dos participantes deste estudo teve sua formação anterior à implementação desses programas, os mesmos encontraram nos cursos de pós-graduação uma maneira de superar a defasagem da formação recebida. Os cursos de especialização foram realizados após ingressarem na área da saúde, pois conforme os discursos, não tiveram uma formação que os instrumentalizassem teórica e metodologicamente para uma atuação nos serviços de saúde: “depois que nós entramos no hospital X, que nós fomos correr atrás de formação que ainda é muito pouca” (Priscila).

Barata, Dias e Dias (2011), ao fazerem uma análise da integração do serviço-escola na formação dos profissionais de saúde, ressaltam que a formação baseada no modelo assistencial biomédico, faz com que os recém-formados ao entrarem nos serviços de saúde, procurem por práticas educativas ou ações de educação permanente por não se adequarem às reais demandas do mundo do trabalho. Assim, os profissionais tiveram que investir em formação para trabalhar no âmbito da saúde, seja através de cursos de aperfeiçoamento ou de especialização, que lhes capacitassem para atender as demandas da realidade hospitalar.

Entre os cursos de especialização mais procurados pelos entrevistados encontra-se primeiramente a formação em Psicologia Hospitalar, seguida pela Psicologia Médica e Psico-Oncologia. Além da procura por uma formação que os instrumentalizasse nesse novo campo, os entrevistados buscaram também nas diferentes teorias psicológicas que lhes dessem suporte teórico para sua práxis. Dentre as várias teorias psicológicas, a Psicanálise é a que tem embasado o fazer da maioria dos entrevistados, seguida pela abordagem Sistêmica e pelas perspectivas Comportamentais.

Em pesquisa realizada com psicólogos que atuam nos hospitais da Região Metropolitana de Florianópolis, Marcon, Luna e Lisbôa (2004), observaram que dentre as perspectivas teóricas utilizadas pelos profissionais a Psicanálise predomina, seguida pela perspectiva Cognitivo-Comportamental e pela abordagem Sistêmica. No Rio Grande do Norte, a Psicanálise também é a mais utilizada pelos psicólogos hospitalares, seguida pela teoria Comportamental (Yamamoto, Trindade \& Oliveira, 2002).

\section{O cotidiano do psicólogo no hospital}

Apesar das diferentes abordagens utilizadas, a forma de trabalho entre os entrevistados apresenta-se semelhante. Todos realizam atendimento psicológico individual, seja no leito, na radioterapia, na quimioterapia ou no ambulatório, como a principal forma de intervenção no âmbito hospitalar.

Em pesquisa sobre a formação e a prática profissional dos psicólogos que trabalham no SUS, Spink (2006), ressalta que $42,1 \%$ dos entrevistados apresentam o atendimento individual como a principal forma de intervenção, corroborando os dados encontrados neste estudo. A área da saúde, até pouco tempo, ainda era sinônimo de clínica tradicional, se caracterizando como uma atividade centrada no indivíduo com objetivos psicoterapêuticos e psicodiagnósticos (Romagnoli, 2011). Os psicólogos se sentem mais seguros quando realizam atendimentos clínicos individualizados, uma vez que sua formação foi direcionada para o atendimento clínico em consultório, o que os deixam sem ferramentas teóricas, técnicas e críticas para atuar no SUS (Dimenstein, 2001; Romagnoli, 2011).

Os psicólogos entrevistados têm percebido que nem todas as técnicas realizadas nos consultórios são aplicáveis ao ambiente hospitalar. A grande demanda por atendimento e o baixo número de profissionais, as dificuldades para se conseguir a privacidade para os atendimentos e a perspectiva de encontro único com o paciente, exigem que o psicólogo atuante no campo da saúde, reinvente sua intervenção e construa novos fazeres.

Conforme ressalta Ferreira Neto (2010a), por muito tempo a formação em Psicologia no Brasil esteve alicerçada numa concepção clássica de clínica orientada pelo agir médico, marcada pelas atividades de 
psicoterapia e/ou psicodiagnóstico realizados nos consultórios particulares, numa perspectiva intraindividual. A partir da década de 1980, tendo em vista o contexto sócio-histórico e político brasileiro, marcado por importantes reivindicações sociais, a formação em Psicologia passa a incorporar em seus debates a dimensão social, levando a um rompimento com o modelo anterior e a ressignificação das práticas profissionais (Ferreira Neto, 2010b). Desse modo, o psicólogo se vê diante dos desafios de construir novos modos de agir, bem como dialogar com profissionais e teorias de outros campos do conhecimento (Ferreira Neto, 2010a).

Inserido no hospital, o profissional da psicologia deve se pautar em técnicas que enfoquem não somente o atendimento em nível individual, mas também o grupal visando a intervenção em um número maior de sujeitos: "É uma intervenção muito boa, grupos no hospital, para esse público aqui, que é o paciente que chega muito desinformado, tudo que ele escuta no corredor o abala" (Priscila).

Os grupos de familiares e pacientes com a mesma patologia, como é o caso dos pacientes oncológicos, permite através da troca de experiências, uma maior identificação com o sofrimento emocional, possibilitando auxílio mútuo no enfrentamento do adoecimento e na motivação necessária para a aceitação e prosseguimento do tratamento (Ismael \& Oliveira, 2008; Mello Filho, 2000). O atendimento em grupo, ao abranger um número maior de pessoas, além de trazer bons resultados para os pacientes, também se apresenta como uma alternativa de tratamento mais viável economicamente, se adéqua às altas demandas de atendimento presentes nas instituições públicas e ainda encontra certa resistência ao individualismo exacerbado da sociedade contemporânea (Romagnoli, 2011; Venâncio, 2004).

Para Venâncio (2004), quanto mais informado o paciente estiver de sua doença, maior será sua capacidade de enfrentar este momento, além de ter uma maior confiança na equipe. Dessa forma, ações de educação em saúde estão sendo realizadas pelos psicólogos da rede oncológica de Belo Horizonte, como uma intervenção que visa auxiliar os pacientes na busca por informações.

Eu acho que a questão da informação talvez seja muito produtiva, porque, às vezes a pessoa não tem informação sobre aquilo, sobre o que é a doença. Às vezes, não pergunta na hora da consulta. [...] Às vezes, vai embora, sem saber direito o que é aquilo [...]. (Poliana)
A literatura tem apontado que as intervenções psicoeducativas no contexto hospitalar auxiliam na minimização da ansiedade, na adesão tanto do paciente como de seus familiares ao tratamento, além de os prepararem para as dificuldades que poderão encontrar durante o processo de tratamento (Ismael \& Oliveira, 2008). A orientação ao paciente pode abordar desde o incentivo para a procura dos demais profissionais de saúde para melhor lhe darem informações, como também orientações breves sobre o tratamento (Gouveia \& Prade, 2008).

Venâncio (2004) ressalta que os psicólogos precisam oferecer informações em linguagem acessível ao paciente, bem como verificar se as informações fornecidas pela equipe de saúde foram compreendidas, o que corrobora os dados encontrados. Outro ponto destacado pelos entrevistados diz respeito aos conhecimentos específicos de cada profissional. Assim, o psicólogo deve orientar o paciente dentro de sua área, possibilitando e estimulando a procura por informações com outros profissionais:

[...] o trabalho do psicólogo também é orientar [...]. Eu vou orientar até onde me cabe e vou até reforçar esse paciente a buscar uma orientação do médico. Eu nunca vou orientar em termos médicos [...] Eu acho que informar, nem sempre os médicos tem tempo pra isso [...] Então, eu acho importante a gente ter esse foco na educação também. [...] Então eu acho que isso só veio melhorar, e falar em saúde, educação em saúde. Eu acho que é fundamental em vários contextos e o psicólogo pode atuar. (Priscila)

E aqui a gente, às vezes, atua é orientando mesmo [...]. Então, eles precisam de informação mesmo. É de alguém que possa escutá-lo e de conversar no mesmo nível, porque são pacientes que chegam aqui com o vocabulário pobre. [...] Às vezes, a gente precisa sim, de orientar e de trabalhar, nessa promoção da saúde sim [...]. (Paula)

As estratégias de orientação e informação aos pacientes e familiares mais utilizados pelos entrevistados, foram através dos atendimentos individuais ou nos grupos terapêuticos. Cartilhas informativas têm sido utilizadas, mas em outros setores do hospital, não havendo ainda foco na oncologia: "A gente tem cartilha informativa, cartilhas que orientam, né? [...] especifico da oncologia não. Tem da unidade coronariana, do CTI, tem da cardiopediatria." (Pietra) 


\section{Dificuldades encontradas pelos psicólogos para a realização de sua práxis...}

As intervenções em grupo são ressaltadas, tanto pela literatura como pelos entrevistados, como eficazes na instituição hospitalar. No entanto, limites institucionais como falta de espaço físico, as demandas cotidianas e o baixo número de psicólogos nas instituições hospitalares contribuem para que a atuação desse profissional fique restrita aos atendimentos individuais: "[...] já teve grupos na sala de espera da radioterapia, porém foi uma questão mesmo de falta de infraestrutura mesmo, porque o hospital vem passando por reformas constantes e frequentes, então a gente tem muita dificuldade." (Paula)

Na oncologia a gente, por um tempo, fez atendimento de sala de espera. Eram quatro profissões... fazia a sala de espera, em relação à educação, participação delas, a motivação ao autocuidado [...] mas foi por um período [...] a mastologia ficou sem lugar durante dois anos. [...] vamos ver se vai voltar. (Pedro)

Além da falta de espaço para a realização dos grupos, os entrevistados apontam para a diferença do público atendido no hospital como um fator que viabiliza ou não a construção dos grupos terapêuticos. Para os psicólogos, hospitais públicos que têm seu atendimento voltado para o câncer, possibilitam intervenções grupais: "[...] Esse é um tipo de intervenção muito legal no hospital, pra esse foco que é o nosso (oncologia). Talvez para um outro hospital, por exemplo o grupo não funcionaria, até porque é um público diferente" (Priscila). Já os hospitais particulares que não apresentam como foco específico a oncologia o trabalho com grupos pode ficar inviabilizado:

Como eu tenho um hospital particular. E com paciente particular e convênio, o paciente ele não fica muito tempo internado, geralmente quando ele tá internado ela tá em apartamento. A própria quimioterapia eu tenho menos leitos do que eu tinha, por exemplo, no $Y$ [hospital público]. Então, eu não consigo assim um número de pacientes para a realização de grupos [...] a própria estrutura hospitalar, ela não viabiliza a questão do grupo. (Patrícia)

A utilização de materiais educativos pelos psicólogos, também apresenta dificuldades. Rozemberg, Silva e Vasconcellos-Silva (2002), apontam que a construção dos impressos hospitalares, centra-se mais no que os profissionais de saúde identificam como informações importantes a serem passadas para os usuários.
Nesse sentido, os materiais apresentam uma escrita profissional, que pode não ser compreendida pelos pacientes. Uma das alternativas para a superação da dificuldade de comunicação presente nos materiais é apontada por Pedro ao sugerir que juntamente com a cartilha informativa há a necessidade de orientações dos profissionais:

[...] algum profissional de saúde que pudesse tá na sala de espera, explicar esse livretinho e distribuir para cada paciente [...] porque ele [paciente] sai de lá informado, sai de lá com um manual que ele começa a saber onde procurar, o que fazer e tal. (Pedro)

Os psicólogos participantes desse estudo enfatizam que se deve ter cuidado ao utilizar materiais impressos na orientação aos pacientes, pois o material por si só, embora importante, não é suficiente:

O material é muito importante sim, mas ele é insuficiente se não há uma abertura das próprias pessoas de querer absorver isso. Quantas cartilhas, quantas coisas que as pessoas, e até mesmo livros que as pessoas compram, compram, mas não lê e se lê também não absorve né [...]. (Paloma)

Mesmo se configurando como uma importante estratégia para o trabalho junto aos pacientes oncológicos, Freemantle et al. (2000) concluem que os materiais impressos apresentam resultados pouco expressivos, mesmo quando estratégias de comunicação, como palestras e workshops, são utilizados em conjunto. $\mathrm{O}$ diálogo e a compreensão dos diferentes saberes e práticas, tem sido considerados como elementos fundamentais em qualquer processo educativo (Acioli, 2008). A escuta oferecida pelo psicólogo aos pacientes faz-se importante, pois está capacitado a promover uma escuta diferenciada dos significados do processo de adoecimento-tratamento (Henoch \& Danielson, 2009). É importante ressaltar, que o processo educativo e a compreensão dos valores e sentidos que o paciente tem de sua saúde e doença, objetivam desenvolver sua autonomia em busca de uma qualidade de vida.

Trabalhar em hospital, com pacientes que possuem uma doença crônica e carregam consigo todo um imaginário social de sofrimento, deterioração e morte, exige que os psicólogos tenham uma formação acadêmica de qualidade para que seu trabalho possa atender as demandas que lhe são dirigidas com ética e capacidade profissional. As políticas públicas educacionais e de saúde têm buscado o encontro do universo 
teórico das universidades com o mundo real dos serviços de saúde. Espera-se que tais iniciativas possam formar profissionais mais próximos da realidade do sistema de saúde vigente no país.

Apesar da distância entre teoria e prática em sua formação, os entrevistados têm buscado novas formas de atuar em um ambiente diferente daquele conhecido na graduação. No dia a dia, intervenções tiveram que ser adaptadas à instituição e à clientela atendida pelo hospital. Assim, além dos atendimentos individuais, grupos e ações de educação em saúde foram sendo construídos e reconstruídos a cada momento.

Os caminhos encontrados pelos psicólogos apontam para tentativas de superações das deficiências e limitações presentes em sua práxis. O psicólogo tem construído um campo de atuação que considera as dimensões subjetiva e psicossocial, estreitando diálogos com outros campos de conhecimento, na perspectiva da interdisciplinaridade.

\section{REFERÊNCIAS}

Acioli, S. (2008). A prática educativa como expressão do cuidado em saúde pública. Revista Brasileira de Enfermagem, 6, 117121.

Andreoli, P. B. A. (2008). Psicologia no hospital e os caminhos para a assistência na UTI. In E. Knobel, P. B. A. Andreoli \& M. R. Erichman (Eds.), Psicologia e humanização: Assistência aos pacientes graves (pp. 3-12). São Paulo: Atheneu.

Angerami, C. V. A. (2001). E a psicologia entrou no hospital. São Paulo: Pioneira.

Barata, J. M. L., Dias, C., \& Dias, P. S. (2011). Ressignificando a formação: Integração serviço-escola, um diálogo necessário em saúde. In L. Kind, C. B. Batista \& L. Gonçalves (Eds.), Universidade e serviços de saúde: Interfaces, desafios e possibilidades na formação profissional em saúde (pp.100-110). Belo Horizonte: Editora PUC Minas.

Barbosa, L. N. F., \& Francisco, A. L. (2007). A subjetividade do câncer na cultura: Implicações na clínica contemporânea. Revista SBPH, 10, 9-24.

Bardin, L. (1977). Análise de conteúdo (L. A. Reto e A. Pinheiro, Trans.). Lisboa: Edições 70.

Bastos, A. V. B., Gondim, S. M. G., \& Borges-Andrade, J. E. (2010). O psicólogo brasileiro: Sua atuação e formação profissional. O que mudou nestas últimas décadas? In Yamamoto, $\mathrm{O}$. H., \& A. L. F. Costa, (Eds.) Escritos sobre a profissão de psicólogo no Brasil (pp. 255-270). Natal: EDUFRN.

Batista, C. B., \& Gonçalves, L. (2011). Marcos sobre a integração ensino-serviço na formação de profissionais para a saúde. In L. Kind, C. B. Batista \& L. Gonçalves (Eds.), Universidade e serviços de saúde: Interfaces, desafios e possibilidades na formação profissional em saúde (pp. 25-48). Belo Horizonte: Editora PUC Minas.

Brasil. Ministério da Saúde. (1996). Conselho Nacional de Saúde. Resolução 196 de 10 de outubro de 1996. Diretrizes e normas regulamentadoras de pesquisas envolvendo seres humanos. Brasília: Ministério da Saúde.
Brasil (1998). Lei $n^{o} 3535$ de 02 de setembro de 1998. Dispõe sobre a reorganização, reorientação e acompanhamento da assistência oncológica no Brasil. Brasília: Ministério da Saúde.

Brasil (2007). Ministério da Educação. Ministério da Saúde. Programa Nacional de Reorientação da Formação profissional em Saúde-Pró-Saúde. Brasília: Ministério da Educação.

Brasil (2008). Portaria Interministerial $n^{\circ} 1.802$, de 26 de agosto de 2008. Institui o Programa de Educação pelo trabalho para a Saúde - PET-Saúde. Brasília.

Carvalho, Y. M., \& Ceccim, R. B. (2006). Formação e educação em saúde: Aprendizados com a saúde coletiva. In G. W. Campos, M. C. S. Minayo, M. Akerman, M. Drumond Júnior \& Y. M. Carvalho (Eds.), Tratado de saúde coletiva (pp. 149-182). Rio de Janeiro: Fiocruz.

Castro, A. F., \& Yamamoto, O. H. (1998). A psicologia como profissão feminina: Apontamentos para estudo. Estudos de Psicologia, 3, 147-158.

Conselho Federal de Psicologia (2010). Atuação dos psicólogos nos serviços hospitalares do Sistema Único de Saúde - SUS: Relatório descritivo preliminar de pesquisa. Brasília: Conselho Federal de Psicologia.

Dimenstein, M. (2001). O psicólogo e o compromisso social no contexto da saúde coletiva. Psicologia em Estudo, 6, 57-63.

Dóro, M. P., Pasquin, R., Medeiros, C. R., Bitencourt, M. A., \& Moura, G. L. (2004). O câncer e sua representação simbólica. Psicologia: Ciência e Profissão, 24, 120-134.

Ferreira Neto, J. (2010a). Uma genealogia da formação do psicólogo brasileiro. Memorandum 18,130-142.

Ferreira Neto, J. (2010b) A atuação do psicólogo no SUS: Análise de alguns impasses. Psicologia: Ciência e Profissão, 30, 390403.

Feuerwerker, L. C. M. (2003). Educação dos profissionais de saúde hoje: Problemas, desafios, perspectivas e as propostas do Ministério da Saúde. Revista da ABENO, 3, 24-27.

Flick, U. (2009). Introdução à pesquisa qualitativa ( $3^{\mathrm{a}}$ ed) (J. E. Costa, Trad.). Porto Alegre: Artmed.

Freemantle N., Harvey E. L., Wolf F., Grimshaw J. M., Grili R., $\&$ Bero L. A. (2000). Printed educational materials: Effects on professional practice and health care outcomes. Cochrane Database of Systematic Reviews. Retirado de http://www. ncbi.nlm.nih.gov/pubmed/10796502?dopt=Abstract\&holding=f 1000,f1000m,isrctn

Gouveia, P. A. R., \& Prade, C. V. (2008). Intervenção neuropsicológica do paciente vítima de acidente vascular cerebral. In E. Knobel, P. B. A. Andreoli \& M. R. Erichman (Eds.) Psicologia e humanização: Assistência aos pacientes graves (pp. 21-37). São Paulo: Atheneu.

Guareschi, N. M. F., Dhein, G., Reis, C., Machry, D. S., \& Bennemann, T. (2010). A formação em psicologia e a inserção para o trabalho no Sistema Único de Saúde. In N. M. F. Guareschi, A. Scisleski, C. Reis, G. Dhein \& M. A. Azambuja (Eds.) Psicologia, formação, política e produção em saúde (pp. 54-98). Porto Alegre: EDIPUCRS.

Henoch, I. \& Danielson, E. (2009). Existential concerns among patients with cancer and interventions to meet them: An integrative literature review. Psycho-Oncology, 18, 225-23e.

Instituto Nacional do Câncer - INCA (2011). Estimativa 2012: Incidência de câncer no Brasil. Rio de Janeiro: Instituto Nacional do Câncer. 
Ismael, S. M. C., \& Oliveira, M. F. P. (2008). Intervenção psicológica na clínica cirúrgica. In E. Knobel, P. B. A. Andreoli \& M. R. Erichman (Eds.), Psicologia e humanização: Assistência aos pacientes graves (pp. 83-91). São Paulo: Atheneu

Junqueira, M. H., \& Kovács M. J. (2008). Alunos de psicologia e educação para a morte. Psicologia: Ciência e Profissão, 28, 506-519.

Karam, C. H., Guimaro, M. S., \& Rodrigues, R. T. S. (2008). Abordagem psicológica do paciente submetido a transplante de órgãos. In E. Knobel, P. B. A. Andreoli \& M. R. Erichman (Eds.), Psicologia e humanização: Assistência aos pacientes graves (pp. 65-81). São Paulo: Atheneu

Kind, L., \& Coimbra, J. R. (2011). Revezamentos entre teoria e prática na reorientação da formação em saúde. In L. Kind, C. B. Batista \& L. Gonçalves (Eds.), Universidade e serviços de saúde: Interfaces, desafios e possibilidades na formação profissional em saúde (pp. 68-99). Belo Horizonte: Editora PUC Minas.

Kóvacs, M. J., Macieira, R. C., \& Carvalho, V. A. (2008). Formação profissional em psico-oncologia. In V. A. Carvalho et al. (Orgs.), Temas em psico-oncologia (pp. 546-555). São Paulo: Summus.

Marcon, C., Luna, I. J., \& Lisbôa, M. L. (2004). O psicólogo nas instituições hospitalares: Características e desafios. Psicologia: Ciência e Profissão, 24, 28-35.

Mello Filho, J. (2000). Grupo e corpo: Psicoterapia de grupo com pacientes somáticos. São Paulo: Artmed.

Ministério da Saúde. Secretaria de Atenção à Saúde. Cadastro Nacional de Estabelecimentos de Saúde. Retirado de http:// cnes. datasus.gov.br

Rey, F. L. G. (2006). As representações sociais como produção subjetiva: Seu impacto na hipertensão e no câncer. Psicologia: Teoria e Prática, 8, 69-85.

Romagnoli, R. C. (2006). A formação dos psicólogos e a saúde pública. Pesquisas e Práticas Psicossociais, 1, 1-14.

Romagnoli, R. C. (2011). Reflexões acerca da formação dos psicólogos e sua relação com a saúde pública. In L. Kind, C. B. Batista \& L. Gonçalves (Eds.), Universidade e serviços de saúde: Interfaces, desafios e possibilidades na formação profissional em saúde (pp. 111-128). Belo Horizonte: Editora PUC Minas.

Rozemberg, B., Silva, A. P. P., \& Vasconcellos-Silva, P. R. (2002). Impressos hospitalares e a dinâmica de construção de seus sentidos: O ponto de vista dos profissionais de saúde. $\mathrm{Ca}$ derno de Saúde Pública, 18, 1685-1694.
Rudnicki, T., \& Carlotto, M. S. (2007). Formação de estudante da área da saúde: Reflexões sobre a prática de estágio. Revista SBPH, 10, 97-110.

Santos, M. A. (2003). Perto da dor do outro, cortejando a própria insanidade: O profissional de saúde e a morte. Revista da SPAGESP, 4, 12-24.

Silva, R. C. (1992). A formação em psicologia para o trabalho na saúde pública. In F. C. B. Campos (Ed.), Psicologia e saúde: Repensando práticas (pp. 25-40). São Paulo: Hucitec.

Silva, R. C. (2010). A formação em psicologia para o trabalho na saúde pública. In F. C. B. Campos \& M. J. Spink (Eds.), Psicologia e saúde: Repensando práticas (2 $2^{\mathrm{a}}$ ed.) (pp. 31-51). São Paulo: Hucitec.

Silva, L. C. (2006). O sentido do cuidado na vivência da pessoa com câncer: Uma compreensão fenomenológica (Tese de doutorado). Universidade de São Paulo, São Paulo.

Silva, L. C. (2009). O sofrimento psicológico dos profissionais de saúde na atenção ao paciente com câncer. Psicologia para América Latina, 16, s/p.

Spink, M. J. (2003). Psicologia social e saúde: Práticas, saberes e sentidos ( $7^{\mathrm{a}}$ ed.) Petrópolis, RJ: Vozes.

Spink, M. J., Bernardes, J. S., \& Menegon, V. S. M. (2006). A psicologia em diálogo com o SUS: Prática profissional e produção acadêmica. Brasília: ABEP.

Teixeira, L. A., \& Fonseca, C. O. (2007). Sobre a história social do câncer. In L. A. Teixeira e C. O. Fonseca (Eds.), De doença desconhecida a problema de saúde pública: o INCA e o controle do câncer no Brasil (pp.13-24). Rio de Janeiro: Ministério da Saúde.

Turato, E. R. (2005). Métodos qualitativos e quantitativos na área da saúde: Definições, diferenças e seus objetos de pesquisa. Revista de Saúde Pública, 39, 507-514.

Venâncio, J. L. (2004). Importância da atuação do psicólogo no tratamento de mulheres com câncer de mama. Revista Brasileira de Cancerologia, 50, 55-63.

Yamamoto, O. H., Trindade, L. C. B. O., \& Oliveira, I. F. (2002). O psicólogo em hospitais do Rio Grande do Norte. Psicologia USP , 13, 217-246.

World Health Organization. (2009). World Cancer Report, 2008. Lyon: International Agency for Research on Cancer.

Recebido: 06/02/2012

Última revisão: 24/11/2012

Aceito: 05/12/2012 